\title{
L'EXAMEN DES ALCOOLS AMYLIQUES POUR GERBER
}

\author{
par \\ P. JAMOTTE \\ Station laitière de Gembloux (Belgique)
}

L'alcool amylique est un des réactifs utilisés dans les méthodes acido-butyrométriques de détermination de la matière grasse dans les produits laitiers (laits, crèmes, fromages, etc.).

C'est à Gerber que revient le mérite d'avoir découvert le moyen de faciliter le rassemblement de la matière grasse en une seule centrifugation par l'adjonction d'alcools gras et notamment d'alcool amylique.

L'alcool amylique intervient à la dose d'environ 5 p. 100 dans les déterminations butyrométriques.

L'alcool amylique, utilisé pour le Gerber, réagit chimiquement en présence du produit laitier et de l'acide sulfurique. Cette double réaction entraîne l'apparition de traces de produits formés, causes de surcharges dans la couche grasse séparée. Ces dernières sont compensées par des causes de réductions dues à l'entrainement, dans la phase aqueuse, de substances grasses ou dérivées.

Compte tenu de ces phénomènes, il importe que l'alcool amylique employé présente des caractéristiques bien définies en vue de convenir lors des déterminations acido-butyrométriques.

\section{Qualités requises de l'alcool amylique pour Gerber}

Jusqu'à présent, nous ne disposons, en Belgique, que de deux textes de référence précisant les qualités requises pour les alcools amyliques.

Le plus ancien a été publié par la Commission consultative des denrées alimentaires (1). Il ne retient, comme spécification, que ce qui suit : "alcool amylique vérifié dans un essai à blanc - P. S. $\pm 0,815 \mathrm{p}^{t}$ éb. : $128-132^{\circ} \mathrm{C} \%$.

Un autre texte plus détaillé a été repris dès 1949 et 1950 dans les normes belges NBN 200 et 209 se rapportant aux dosages de la matière grasse dans les crèmes $[2,3]$.

"Alcool amylique - La masse spécifique à $20^{\circ} \mathrm{C}$ de l'alcool

(1) Dans le texte, la dénomination "alcool amylique " couvre en réalité un produit complexe, composé d'isomères et de produits annexes, convenant pour le Gerber. 
amylique utilisé est égale à $0,811 \pm 0,002 \mathrm{~g} / \mathrm{ml}$; son point d'ébullition est tel que $95 \mathrm{p} .100 \mathrm{du}$ produit distillent entre 128 et $132^{\circ} \mathrm{C}$.

L'alcool amylique est constitué du mélange chimiquement pur de deux isomères du pentanol, c'est à-dire de :

$$
\text { méthyle_2 - butanol } 1 \text { et de méthyl } 3 \text { - butanol } 1
$$

Il ne peut renfermer ni eau, ni acides, ni furfurol, ni autres substances organiques. Sa solubilité est telle que lorsqu'on mélange $10 \mathrm{ml}$ d'alcool amylique avec $10 \mathrm{ml}$ d'acide chlorhydrique concentré $(1,156)$ il en résulte une solution claire qui se scinde en deux phases après addition de $1 \mathrm{ml}$ d'eau. "

Ces teux textes, auxquels on doit se référer, en Belgique, pour effectuer un contrôle d'alcool amylique pour Gerber sont, à notre avis, ou incomplets, ou trop peu précis et, à certains égards, trop sévères.

La littérature spécialisée étrangère fournit beaucoup de prescriptions sur le sujet. De nombreuses définitions de l'alcool amylique convenant pour le Gerber ont été publiées et recopiées [4, 5, 6, 7].

De nombreux chercheurs ont décrit divers tests visant à reconnaître un alcool amylique convenable $[8,9,10,11,12,13]$.

Cependant, certaines prescriptions reprises dans les normes de pays voisins s'avèrent, en réalité, difficiles à satisfaire.

Dans les dernières années, la chromatographie en phase gazeuse a permis un examen rapide et détaillé de certaines caractéristiques des alcools amyliques. Ce procédé moderne d'analyse présente un réel intérêt et facilite grandement les contrôles. Toutefois les jugements portés sur la convenance d'un alcool amylique basés sur ce seul procédé ne sont pas suffisants.

Il serait simple d'exiger pour le Gerber un alcool amylique d'une grande pureté. Mais il ne faut pas perdre de vue le côté pratique du problème.

Les méthodes acido-butyrométriques sont et restent, avant tout, des méthodes industrielles rapides de dosage des matière grasses. Dès lors, l'emploi d'un réactif coûteux est à exclure. On ne peut donc exiger d'un alcool amylique usuel (vendu à 40-50 F. B. le l) des caractéristiques de pureté semblables à celles requises pour un alcool amylique pour analyse $( \pm 500 \mathrm{~F}$. B. le 1). Le degré de précision des méthodes acido-butyrométriques n'est d'illeurs pas très élevé : une erreur de l'ordre de 1,5 à 2,5 p. 100 selon le produit analysé, est logiquement admise par les praticiens. L'emploi d'un alcool amylique de qualité courante (chimiquement pur dit la NBN) se justifie et il devient très théorique d'émettre des exigences anormalement élevées telles que à certains points de vue des alcools de qualité pour analyse (p. a.) seraient à proscrire.

Ainsi, la NBN belge considère que l'alcool amylique, constitué du mélange de deux isomères ne peut renfermer ni eau, ni acides, 
ni furfurol ni autres substances organiques. Ces exigences sont rarement satisfaites en pratique, plus particulièrement en ce qui concerne le dernier terme de la phrase.

Voici d'ailleurs quelques résultats d'analyse chromatographique de divers alcools amyliques d'origines belges ou étrangères.

Analyse chromatographique d'échantillons

\begin{tabular}{|c|c|c|c|c|c|c|c|c|}
\hline 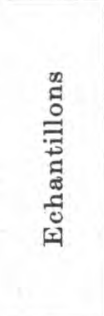 & 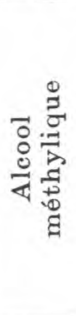 & 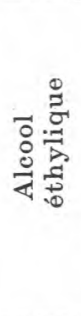 & 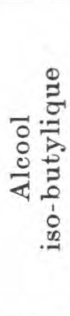 & 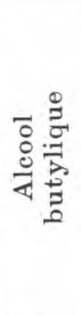 & 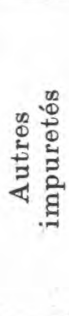 & 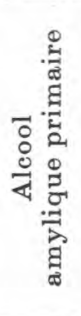 & 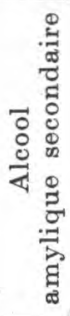 & 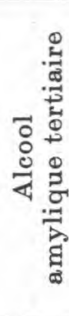 \\
\hline B 1 & 0,2 & 0,2 & 3,5 & 0,3 & 0,1 & 95,7 & - & - \\
\hline B 2 & 1,3 & 2,4 & 2,8 & 0,5 & 0,3 & 92,5 & - & - \\
\hline B345 & 0,5 & 0,7 & 2,0 & 0,2 & 0,1 & 96,3 & - & - \\
\hline B 6 & 1,4 & 2,7 & 3,0 & 0,5 & 0,6 & 91,6 & - & - \\
\hline D 1 & 1,6 & 3,3 & 3,1 & 0,6 & 0,6 & 90,7 & - & - \\
\hline D 2 & 2,1 & 4,2 & 4,0 & 0,8 & 0,8 & 88,0 & - & - \\
\hline D 3 & 0,6 & 0,8 & 3,4 & 0,3 & 0,1 & 94,7 & - & - \\
\hline V & - & - & 3,2 & 0,4 & 0,8 & 95,6 & - & - \\
\hline B & - & - & 0,2 & - & - & 95,5 & - & 4,1 \\
\hline B & - & - & 1,3 & 0,2 & 0,9 & 97,3 & - & - \\
\hline B & 一 & - & 0,2 & - & 0,6 & 98,0 & - & 1,4 \\
\hline F & - & - & - & - & 0,5 & 99,3 & - & - \\
\hline $\mathrm{CHF}$ & 0,1 & - & 0,9 & 0,2 & - & 98,8 & - & - \\
\hline $\mathrm{CHS}$ & - & - & 0,2 & 0,1 & 0,1 & 99,5 & - & - \\
\hline $\mathrm{N}_{2}$ & - & - & 1,0 & 0,1 & 4,1 & 95,0 & - & - \\
\hline
\end{tabular}

A rapprocher de ces résultats obtenus à Gembloux, ceux publiés par la Station laitière d'Hillerød [9].

\begin{tabular}{|c|c|c|c|c|c|c|}
\hline & 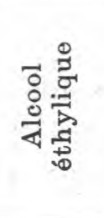 & 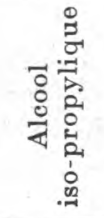 & 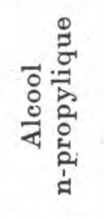 & 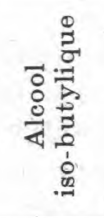 & 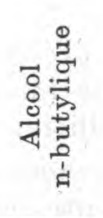 & 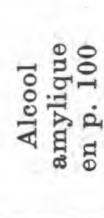 \\
\hline 1 & - & - & - & traces & - & 99,0 \\
\hline 2 & - & - & traces & 11,0 & - & 89,0 \\
\hline 3 & - & - & traces & 12,0 & - & 88,0 \\
\hline 4 & - & - & 3,0 & 9,0 & 1,0 & 87,0 \\
\hline 5 & traces & - & 1,0 & 12,0 & - & 87,0 \\
\hline 6 & - & traces & traces & 16,0 & 19,0 & 65,0 \\
\hline 7 & - & - & - & 12,0 & traces & 88,0 \\
\hline 8 & - & - & - & 2,0 & 1,0 & 97,0 \\
\hline 9 & - & - & - & - & - & 99,0 \\
\hline 10 & - & - & - & traces & traces & 99,0 \\
\hline
\end{tabular}


Nous constatons que les alcools pour Gerber contiennent, à côté d'un pourcentage important mais variable d'alcools amyliques primaires, des pourcentages parfois non négligeables d'autres alcools inférieurs, d'alcools butylique et iso-butylique et de certains alcools tertiaires.

Il est bien admis que la présence d'alcools secondaires ou tertiaires est totalement inopportune. Celle-ci entraîne inévitablement un accroissement du pourcentage de gras déterminé et des difficultés de lecture dues à l'apparition d'un trouble gênant.

Par contre, on peut se poser la question : la présence d'un faible pourcentage d'alcool butylique est-elle vraiment indésirable? Pour doser la matière grasse dans les babeurres, nous utilisons, depuis longtemps, une méthode où l'alcool amylique est remplacé par une quantité double d'alcool butylique.

Cette méthode donne des résultats corrects et évite l'apparition fréquente dans le cas des babeurres d'une couche intermédiaire gênante.

La présence d'alcool butylique dans l'alcool pour Gerber tendrait, selon la littérature, à l'obtention de résultats légèrement plus faibles.

Elle favoriserait également une séparation moins nette entre la phase acide et la couche grasse.

La présence d'eau dans l'alcool amylique est encore assez fréquente. Elle modifie le poids spécifique de l'alcool ainsi que le taux de répartition de l'alcool entre la phase aqueuse et la phase grasse. Elle entraînerait donc, théoriquement, une diminution des résultats de dosage.

La présence d'alcools inférieurs (alcool éthylique par exemple) agirait dans le même sens que celle d'alcool butylique ou d'eau.

Pour apprécier la qualité d'un alcool amylique pour Gerber on a, depuis longtemps, fixé certains critères : poids spécifique, point d'ébullition, caractéristiques de distillation, etc.

Ces exigences visent à définir une certaine composition pour les alcools aptes au Gerber.

\section{Poids spécifique}

Le poids spécifique est un critère uniquement physique. Son mode d'expression varie selon les sources. Les dernières indications cont en effet données en masse volumique.

Admettons, sans vouloir trop de précisions, qu'un alcool amylique pour Gerber doit présenter un poids spécifique de 0,81 à 0,82 . 
Dans le tableau suivant, nous avons rapproché les diverses données disponibles concernant les poids spécifiques des constituants possibles d'un alcool pour Gerber.

Il apparaît d'emblée, que la détermination du poids spécifique ne permet pas la sélection d'un alcool valable. Tout au plus, permet-elle de supposer que l'alcool examiné contient probablement des alcools inférieurs (butyliques, etc.) si la valeur est faible ou bien de l'eau, en proportions anormales, si la valeur est élevée.

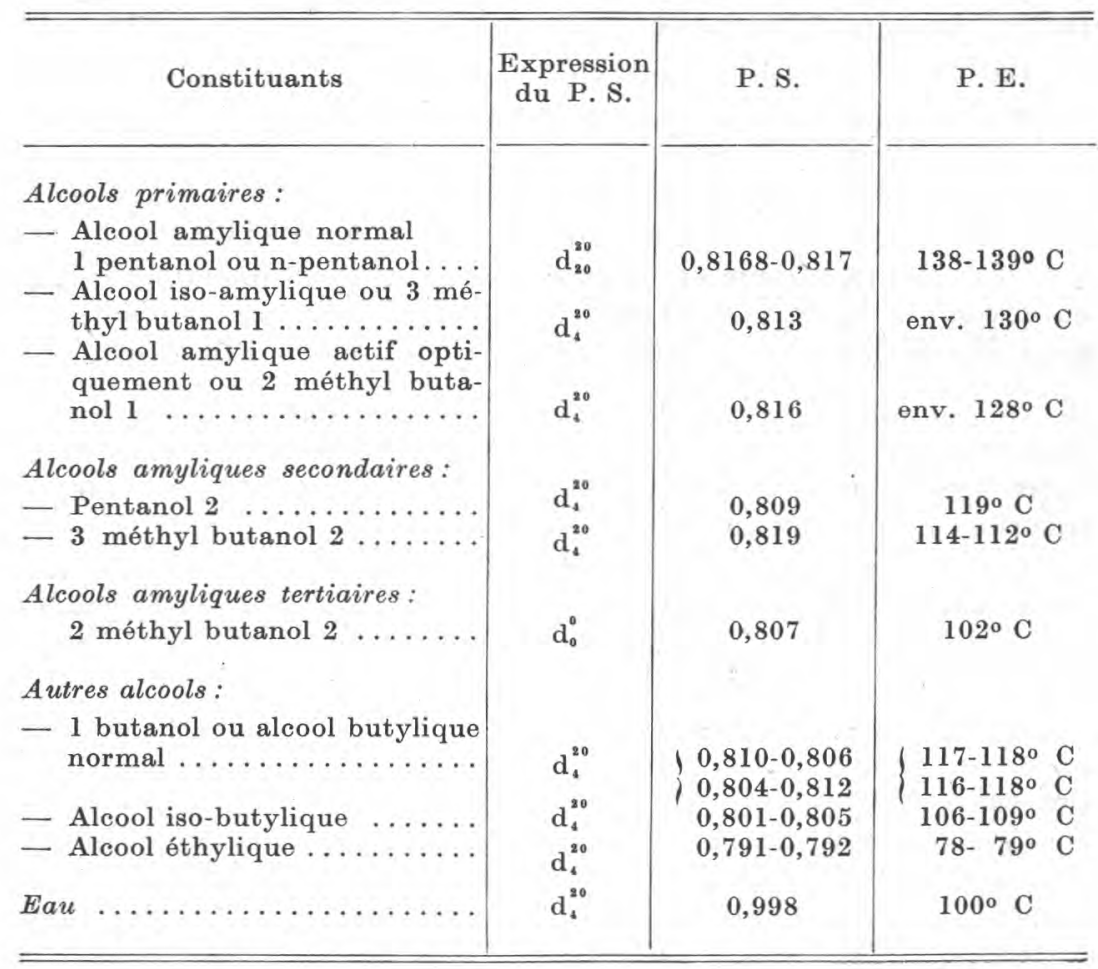

\section{Distillation}

La zone de distillation est un autre critère physique très utilisé dans les définitions d'alcools amyliques pour Gerber.

C'est ainsi que la norme hollandaise NEN 962 reprend à ce sujet les prescriptions suivantes [4] :

"Distillé selon la procédure normalisée NEN 942 (fractions légères) l'alcool amylique doit satisfaire aux exigences suivantes :

“ Début d'ébullition : pas moins de $128^{\circ}$ C. 98 p. $100 \mathrm{du}$ liquide doit distiller en dessous de $132^{\circ} \mathrm{C}$ (tolérance sur la lecture 
des températures $\pm 0,2^{\circ} \mathrm{C}, \mathrm{p}$. E ramené à $760 \mathrm{~mm}$ de mercure, etc.).

"L'efficience de la distillation ne doit pas être moindre que

98 p. 100 (tolérance $\pm 0,2$ p. 100).

"Il ne doit pas rester de résidu après distillation. "

La méthode unifiée allemande de 1963 reprend approximativement les mêmes exigences [5].

Les normes belges NBN 209 et 210 ont été rappelées ci-avant (95 p. 100 entre $128-132^{\circ} \mathrm{C}$ ).

Dans le catalogue d'une firme de produits chimiques, nous avons trouvé l'exigence suivante : 98 p. 100 minimum entre $125^{\circ}$ et $135^{\circ} \mathrm{C}$ (Hopkins-Williams).

Aux Pays-Bas, une nouvelle proposition a été faite récemment. Selon Eisses, les exigences se situeraient comme suit [12] :

10 p. 100 maximum en-dessous de $128^{\circ} \mathrm{C}$ et 98 p. 100 au moins en-dessous de $132^{\circ} \mathrm{C}$, ce qui correspond en fait à l'agrégation possible d'un alcool distillant à raison de 88 p. 100 entre 128 et $132^{\circ} \mathrm{C}$.

De nombreux contrôles d'échantillons d'alcools amyliques pour Gerber nous ont montré que les pourcentages distillant entre 128 et $132^{\circ} \mathrm{C}$ sont, d'une manière générale, moins élevés que ceux requis par les normes précitées.

Le tableau annexé fait foi (X). Il détaille non seulement les critères de distillation, mais aussi les poids spécifiques et la convenance des alcools déterminée par d'autres tests tels que l'essai comparatif, l'essai à blanc ou le test des oléfines.

De l'examen des résultats d'analyses, il ressort que les quelques alcools reconnus mauvais par d'autres tests, présentent également des caractéristiques anormales de distillation.

Cependant, on constate également que de nombreux échantillons valables, passant avec succès les autres tests, présentent des pourcentages distillant entre $128-132^{\circ} \mathrm{C}$ de l'ordre de 70 à $80 \mathrm{p} .100$.

L'inverse est également possible. Ainsi un alcool satisfaisant à raison de 85 p. 100 à la distillation dans la zone admise des températures, apparaît absolument non convenable selon les autres tests et en particulier selon les essais comparatifs.

Il faut aussi faire remarquer que bien des alcools amyliques vendus sans cachet d'usines renommées, comme convenant pour le Gerber, ne satisfont pas du tout aux exigences de distillation reprises dans certaines des normes précitées : ou pourcentage distillant entre $128-132^{\circ} \mathrm{C}: 90$ p. 100,82 p. $100 \ldots$ et même 67,5 p. 100 .

L'épreuve de distillation permet seulement de déceler les alcools contenant des pourcentages particulièrement élevés d'alcools inférieurs ou butyliques. Les points d'ébullition des principales impuretés ont été signalés dans le tableau ci-avant. 
TABLEAU $\mathrm{X}$

Résultats d'analyses

\begin{tabular}{|c|c|c|c|c|c|c|c|c|c|}
\hline \multirow{2}{*}{$\begin{array}{c}\text { Echan- } \\
\text { tillons } \\
\text { ordinaires }\end{array}$} & \multirow{2}{*}{$\begin{array}{c}\text { Poids } \\
\text { spécifiques } \\
20^{\circ} \mathrm{C}\end{array}$} & \multicolumn{6}{|c|}{ Epreuves de distillation } & \multirow{2}{*}{$\begin{array}{c}\text { Essais } \\
\text { à blanc } \\
(\mathrm{p} .100)\end{array}$} & \multirow{2}{*}{$\begin{array}{c}\text { Essais } \\
\text { comparatifs }\end{array}$} \\
\hline & & $\begin{array}{l}\text { Qtés distil. } \\
(\mathrm{ml})\end{array}$ & 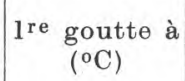 & $\begin{array}{c}<128^{\circ} \mathrm{C} \\
(\text { p. } 100)\end{array}$ & $\begin{array}{c}128^{\circ}-132^{\circ} \mathrm{C} \\
(\mathrm{p} .100)\end{array}$ & $\begin{array}{l}>132^{\circ} \mathrm{C} \\
(\text { p. } 100)\end{array}$ & $\begin{array}{c}\mathrm{p}^{t} \text { sec } \\
\left({ }^{\circ} \mathrm{C}\right)\end{array}$ & & \\
\hline A $40-157$ & 0,817 & 100 & 116 & 41 & 55 & 4 & 158,5 & +0.2 & mauvais \\
\hline 179 & 0,813 & 100 & 114 & 33,5 & 63,5 & 3 & 143 & & bon \\
\hline 182 & 0,812 & 50 & 116 & 8 & 92 & 0 & 129,5 & 0 & bon \\
\hline 188 & 0,813 & 100 & 121 & 44 & 54 & 2 & 135 & traces & mauvais \\
\hline 200 & 0,814 & 100 & - & 28 & 72 & 0 & 130 & 0 & bon \\
\hline $200 b$ & 0,815 & 100 & - & 59 & 39 & 2 & - & traces & mauvais \\
\hline 206 & 0,818 & 50 & 107 & 62 & 38 & 0 & 132 & 0 & - \\
\hline A $41-3$ & 0,814 & 100 & 121,5 & 47 & 53 & 0 & 144,5 & 0 & - \\
\hline 10 & 0,815 & 100 & 117,5 & 26 & 70 & 4 & 146 & 0 & bon \\
\hline 52 & 0,816 & 200 & 124 & 25 & 72 & 3 & 138 & 0 & bon \\
\hline 80 & 0,816 & 200 & 127,5 & 2 & 92 & 6 & 144 & 0 & bon \\
\hline A $47-137$ & 0,816 & 100 & 123 & 18 & 80 & $1-2$ & 138 & 0 & bon \\
\hline $137 b$ & 0,816 & 100 & 120 & 29 & 70 & $1-1,5$ & 136,5 & 0 & bon \\
\hline A $48-169$ & 0,816 & 100 & 120 & 20 & 77 & 3 & 137 & 0 & bon \\
\hline A $51-122$ & 0,813 & 100 & 124,1 & 10 & 85 & 5 & 147 & 0 & bon \\
\hline \multirow{6}{*}{\multicolumn{2}{|c|}{$\begin{array}{l}\text { Sous-cachet (garantis) } \\
\text { M. } 979 \text { p. a. } \\
\text { M. } 978 \text { p. a. } \\
\text { M. B. p. a. } \\
\text { M. B. } \\
\text { Aux. }\end{array}$}} & & & & & & & & \\
\hline & & 100 & 127 & 10 & 90 & - & 129 & & \\
\hline & & 100 & 114 & 32,5 & 67,5 & - & 131 & & \\
\hline & & 100 & 123 & 18 & 82 & - & 130 & & \\
\hline & & 100 & 122 & 21 & 79 & - & - & & \\
\hline & & 100 & 118,5 & 15 & 53 & 1,5 & 135 & & \\
\hline
\end{tabular}


La réalisation des essais de distillation est assez compliquée. Les conditions de distillation sont à observer rigoureusement pour obtenir des résultats comparables. Il faut toujours opérer avec un matériel standardisé et des quantités définies. La vitesse de distillation joue un grand rôle. Les températures doivent être corrigées en fonction de la pression atmosphérique et de la température ambiante.

L'importance de la prise d'essai est énorme. Ainsi, l'épreuve effectuée sur un même alcool, dans des conditions semblables, donne les deux résultats suivants, selon que d'une part on a opéré sur $100 \mathrm{ml}$ dans un ballon de $100 \mathrm{ml}$ ou d'autre part sur $500 \mathrm{ml}$ dans un ballon de $700 \mathrm{ml}$.

\begin{tabular}{|c|c|c|}
\hline Quantité distillée & 1re goutte à & p. 100 entre $128-132^{\circ} \mathrm{C}$ \\
\hline $\begin{array}{l}100 \mathrm{ml} \\
500 \mathrm{ml}\end{array}$ & $\begin{array}{ll}122^{\circ} & \mathrm{C} \\
127^{\circ} & \mathrm{C}\end{array}$ & $\begin{array}{l}66 \\
96\end{array}$ \\
\hline
\end{tabular}

L'essai de distillation ne peut donc, à notre avis, être considéré comme un test pratique utile vu qu'il demande beaucoup de précisions dans l'exécution et qu'il ne donne pas des renseignements suffisants.

Nous reproduisons ei-dessous les résultats publiés par la Station laitière d'Hiller $\varnothing \mathrm{d}[8]$.

Ils se rapportent à la distillation de 21 échantillons d'alcools amyliques pour Gerber, distillation effectuée avec une prise de $200 \mathrm{ml}$ dans un ballon de $500 \mathrm{ml}$. Les échantillons ont été classés en groupes selon leurs valeurs respectives au vu des résultats de distillation.

\begin{tabular}{|c|c|c|c|c|c|c|}
\hline $\begin{array}{c}N^{0 s} \\
\text { échant. }\end{array}$ & $\begin{array}{c}<120^{\circ} \mathrm{C} \\
\text { p. } 100\end{array}$ & $\begin{array}{c}120-124^{\circ} \mathrm{C} \\
\text { p. } 100\end{array}$ & $\begin{array}{c}124-128^{\circ} \mathrm{C} \\
\text { p. } 100\end{array}$ & $\begin{array}{c}128-130^{\circ} \mathrm{C} \\
\text { p. } 100\end{array}$ & $\begin{array}{c}>130^{\circ} \mathrm{C} \\
\text { p. } 100\end{array}$ & Groupes \\
\hline 14 & 3 & 3 & 8 & 50 & 36 & I \\
\hline 15 & 3 & 2 & 5 & 25 & 65 & I \\
\hline 16 & 1 & 2 & 3 & 15 & 79 & I \\
\hline 18 & 5 & 2 & 12 & 42 & 39 & II \\
\hline 12 & 11 & 4 & 5 & 22 & 58 & II \\
\hline 13 & 10 & 4 & 16 & 17 & 53 & III \\
\hline 17 & 8 & 5 & 14 & 64 & 7 & III \\
\hline 2 & 0 & 8 & 40 & 31 & 21 & IV \\
\hline 3 & 0 & 10 & 31 & 30 & 24 & IV \\
\hline 1 & 13 & 25 & 33 & 18 & 10 & VI \\
\hline 8 & 18 & 24 & 31 & 14 & 13 & VI \\
\hline
\end{tabular}


Les conclusions de l'étude danoise, basées sur l'essai pratique, au Gerber, des diverses portions de distillats, sont que les alcools convenables ne peuvent pas comprendre plus de 15 à 20 p. 100 distillant, dans les conditions de l'essai, en dessous de $128^{\circ} \mathrm{C}$ (soit 85 à 80 p. 100 entre 128 et $132^{\circ} \mathrm{C}$ ).

La distillation peut surtout servir à préparer des échantillonstypes, distillant entre 128 et $132^{\circ} \mathrm{C}$, aptes à la réalisation d'essais comparatifs, après avoir été soumis aux autres tests de pureté. L'essai de distillation peut d'ailleurs être avantageusement remplacé par une chromatographie en phase gazeuse.

\section{Analyse chromatographique en phase gazeuse}

Celle-ci donne, en effet, plus rapidement, des indications bien plus précieuses, déterminant exactement les divers alcools entrant dans la constitution du mélange soumis à l'essai.

Certains résultats de nos analyses chromatographiques ont été cités plus haut. Pour les obtenir, nous avons procédé comme suit : l'alcool amylique pour Gerber peut être injecté tel que dans l'appareil de chromatographie gazeuse à l'aide d'une microseringue.

Les modalités techniques de l'analyse sont les suivantes :

Appareil

: Perkin-Elmer $116 \mathrm{E}$.

Colonne de séparation : K : 15\% Polyéthylène glycol sur Carbowax 1500 long $2 \mathrm{~m}$ diamètre $1 / 4$ pouce

Gaz porteur

Débit

Température

Détecteur

Injection
: hydrogène

: $\pm 60 \mathrm{ml} /$ minute

: 100-101 degrés

: Catharomètre

: 1 à 1,2 microlitre.

Analyse quantitative.

L'aire des pics a été calculée par triangulation.

Le rapport de chaque aire multipliée par le coefficient d'atténuation éventuel, à l'aire totale, est une mesure du pourcentage de la substance à laquelle le pic se rapporte.

Les chromatogrammes indiquent les divers alcools constituant les alcools amyliques pour Gerber. La présence d'alcools amyliques secondaires et tertiaires est également détectée, de même celle de l'eau en pourcentages appréciables.

La chromatographie en phase gazeuse donne done plus de renseignements que la distillation fractionnée. 
Il est, à ce propos, intéressant de mettre en parallèle les résultats obtenus par les 2 méthodes. Cette confrontation confirme l'impression première de ce que la distillation entre 128 et $132^{\circ} \mathrm{C}$, selon les exigences néerlandaises par exemple, est un critère trop sévère.

C'est ainsi qu'un alcool amylique contenant, selon le chromatogramme, 97 p. 100 d'alcool convenable pour Gerber ne donne, à la distillation entre 128 et $132^{\circ} \mathrm{C}$ que $85 \mathrm{p}$. 100 d'alcool valable.

Cet exemple tend de plus à démontrer que ni l'épreuve de distillation, ni l'épreuve chromatographique ne constituent des tests réellement suffisants pour juger de la valeur d'un alcool pour Gerber. Les fluctuations possibles entre les divers résultats, selon les conditions d'essai ou d'interprétation sont également à relever.

La chromatographie en phase gazeuse est un moyen élégant de faire une "scopie " des composants volatils d'un alcool amylique pour Gerber.

Elle donne mieux et plus rapidement que la distillation des indications très utiles pour le contrôle. Mais elle n'est pas suffisante.

A preuve, l'alcool cité ci-dessus (97 p. 100 de valable par chromatographie, 85 p. 100 à la distillation) convient à peine, vu qu'il ne satisfait plus pleinement à d'autres tests (essai à blanc - légères traces de substances grasses - essais comparatifs sur lait 3,213 au lieu de 3,20 p. 100 - substances brunissantes : brun rougeâtre).

Comme la distillation, la chromatographie ne peut déceler les traces de substances grasses, le furfurol et autres souillures organiques. Elles ne détecte que les substances volatilisables dans les conditions thermiques de l'essai.

C'est ainsi que le furfurol, révélateur de produits annexes de fermentation, ne peut normalement apparaître ni dans le chromatogramme tel que nous le développons, ni dans la distillation puisqu'il a un point d'ébullition de 161-162 $\mathrm{C}$.

Aussi faudra-t-il toujours avoir recours à un test chimique pour le détecter.

La présence de furfurol ( $\alpha$-furaldehyde) est définie par l'apparition d'une coloration orangée-rouge dans l'alcool amylique, additionné d'une quantité équivalente d'aniline dissoute en milieu faiblement acide [4].

\section{Substances brunissantes à l'acide sulfurique}

La détection du furfurol et des autres impuretés organiques éventuelles s'effectue en recherchant ce que l'on dénomme couram. ment les " substances brunissantes à l'acide sulfurique ». Un mélange à parties égales d'alcool amylique et l'acide sulfurique pour Gerber ne doit pas donner une coloration plus marquée que le jaune paille. 
Lorsque la coloration devient légèrement brunâtre, il y a excès d'impuretés organiques. Ce test est souvent mentionné comme critère limitatif à l'emploi d'un alcool amylique pour Gerber.

Cependant, nous avons trouvé, à diverses reprises, des alcools amyliques convenant parfaitement (aux essais comparatifs) et donnant avec le test en question des colorations nettement brunâtres.

A notre avis, ce test des "substances brunissantes" ne peut pratiquement pas exclure de l'approbation certains alcools considérés comme convenables selon d'autres épreuves plus probantes.

\begin{tabular}{|c|c|c|c|}
\hline Nos des éch. & Types d'alcools & Colorations & $\begin{array}{l}\text { Valeurs des échantillons } \\
\text { selon essais } \\
\text { comparatifs }\end{array}$ \\
\hline 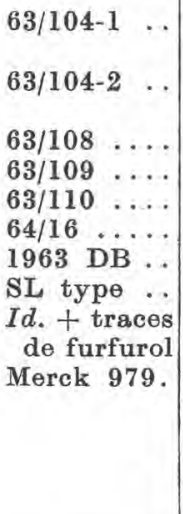 & \begin{tabular}{|} 
alcool amylique pour \\
Gerber \\
\\
$i d$. \\
\\
$i d$. \\
$i d$. \\
$i d$. \\
$i d$. \\
$i d$. \\
$i d$. \\
$i d$. \\
$i d$. \\
alcool éthylique déna- \\
turé à l'éther \\
alcool butylique n. \\
alcool iso-butylique n.
\end{tabular} & $\begin{array}{l}\text { jaunâtre } \\
\text { jaunâtre légèr. } \\
\text { brunâtre } \\
\text { brun très clair } \\
\text { jaune canari } \\
\text { brun clair } \\
\text { brun } \\
\text { jaunâtre } \\
\text { jaunâtre } \\
\text { brun clair } \\
\text { jaune canari } \\
\text { pas de colorat. } \\
\text { jaune canari } \\
\text { jaune }\end{array}$ & $\begin{array}{l}\text { non convenable } 3,25 / 3,11 \\
\text { non convenable } 3,27 / 3,11 \\
\text { non convenable } 4,25 / 3,35 \\
\text { non convenable } 3,29 / 3,19 \\
\text { convenable } \\
\text { convenable } \\
\text { convenable } \\
\text { convenable } \\
\text { convenable } \\
\text { convenable }\end{array}$ \\
\hline
\end{tabular}

\section{Essai à blanc}

Autre essai souvent préconisé, l'essai à blanc consiste, en fait, à effectuer une détermination Gerber dans laquelle le lait est remplacé par une quantité équivalente d'eau. L'alcool satisfait au test lorsqu'aucune trace de matière grasse n'apparaît au sommet de la colonne dans la tige graduée. Un alcool amylique ne satisfaisant pas à l'essai est certainement un mauvais alcool. Mais l'inverse n'est pas établi. Beaucoup d'alcools amyliques, subissant avec succès le test, se révèlent cependant non convenables lors d'autres essais (comparatifs sur laits par exemple).

Les substances grasses ou huileuses, théoriquement détectées par ce test, peuvent très bien ne pas se séparer nettement d'une 
phase acide et aqueuse mais bien être entraînées en surface dans la phase grasse séparée du lait et constituer alors une cause appréciable de surcharge.

Ainsi, l'essai à blanc n'est pas, lui non plus, suffisant.

\section{Test des oléfines}

Un autre test, dit des "oléfines" a également été proposé [11]. Il permet de déceler la présence des alcools amyliques secondaires et tertiaires et de glycérine.

Ce dernier test complète l'essai de distillation ou confirme le test par chromatographie. Il ne met cependant pas en évidence la présence éventuelle de substances grasses.

En conclusion de ce qui précède on peut donc affirmer qu'aucun des tests cités ci-avant, pris seul en considération (P. S. - zone de distillation - chromatographie, furfurol, essai à blanc, essai à l'H2 SO4) ou même à plusieurs, ne peut pleinement satisfaire l'analyste chargé de juger de la valeur d'un alcool.

Seule, la réalisation successive de la plupart des contrôles précités pourrait donner les indications utiles et nécessaires pour se former une opinion valable. Ceci demande done un long travail analytique qui exigera finalement une confirmation par un essai pratique sur lait mettant en parallèle les résultats obtenus au Gerber avec l'alcool soumis aux essais et ceux obtenus par la méthode de référence.

\section{Essais comparatifs sur lait}

Les essais comparatifs constituent les seuls tests pratiques donnant rapidement des indications pleinement satisfaisantes.

Pour autant que les dosages soient effectués correctement (c'est-à-dire en observant minutieusement les modes opératoires requis, en utilisant les réactifs préconisés ainsi que des butyromètres contrôlés et sélectionnés) sur plusieurs échantillons de lait à divers taux de matière grasse, la comparaison entre des résultats obtenus d'une part, au Gerber, (pipette de 10,75 ou 10,77 mal) avec l'alcool à contrôler et d'autre part à la méthode pondérale Röse-Gottlieb, donne assurément les meilleures garanties quant à la valeur du jugement porté. Un alcool amylique convient si les résultats sont concordants.

De plus, comme il n'est guère possible à tous d'effectuer des déterminations selon Röse-Gottlieb (par manque de temps ou de 
moyens), nous pensons qu'il n'y aurait pas d'inconvénients à remplacer cette méthode de référence par un simple Gerber effectué avec le maximum de soins en utilisant un alcool amylique garanti convenable (alcool iso-amylique pour analyse). Cet alcool-type pourrait d'ailleurs être préparé par distillation. Cet étalon serait contrôlé par un laboratoire bien outillé capable d'effectuer tous les tests requis.

Ramenés de la sorte à une simple comparaison entre plusieurs résultats obtenus par acido-butyrométrie, les essais comparatifs sont extrêmement rapides et peu compliqués.

\section{Conclusions}

L'analyse chromatographique, seul procédé à retenir pour la détermination précise de la composition en alcools, peut éventuellement, être omise lors des contrôles courants.

Les essais comparatifs sur laits doivent être considérés comme épreuves suffisantes pour le contrôle des alcools amyliques ordinaires, pour Gerber.

Ils donnent une plus grande certitude que la réalisation laborieuse d'une longue suite d'essais visant à définir des constantes ou des propriétés caractéristiques dont les limites de tolérance sont mal définies. Cet avis rejoint ceux de plusieurs chercheurs étrangers $[13,14]$.

Le processus à suivre pour effectuer les essais comparatifs pourrait être détaillé et codifié.

L'adoption d'une telle procédure simplifierait la définition des caractéristiques physico-chimiques des alcools amyliques pour Gerber.

Celle-ci pourrait alors se réduire à ce qui suit :

Alcool amylique : masse volumique à $20^{\circ} \mathrm{C}: 0,81-0,82 \mathrm{~g} / \mathrm{ml}$

L'alcool amylique doit être constitué d'un mélange chimiquement pur des deux alcools primaires : 3 méthyl-butanol 1 et 2 méthyl-butanol 1 (min. 95 p. 100).

La présence d'autres alcools inférieurs tels que l'alcool butylique (butanol 1) ou iso-butylique (2 méthyl-propanol 1) est tolérée ( $\max .2,5$ p. 100 iso-butanol).

Le taux des impuretés ne peut être tel que l'alcool amylique ne puisse satisfaire à l'essai de conformité ci-dessous : 


\section{Essai de conformité}

Les teneurs en matière grasse de 3 laits à diverses teneurs ( $3 \pm 0,5$ p. 100) sont déterminées, selon Gerber, en utilisant d'une part l'alcool amylique à examiner et d'autre part un alcool amylique pur, composé uniquement des deux constituants principaux précités (éventuellement préparé par distillation).

Les déterminations sont effectuées en triple exemplaire dans des butyromètres sélectionnés (erreur de calibrage de la graduation moindre que $0,03 \mathrm{p} .100)$.

L'écart entre les moyennes des 9 résultats obtenus pour chacune des séries de détermination ne peut atteindre 0,03 p. 100.

\section{Résumé}

Après avoir rappelé les exigences de pureté requises en Belgique et à l'étranger, l'auteur commente les résultats de nombreux contrôles d'alcools amyliques pour Gerber.

Les épreuves de distillation et autres tests couramment proposés ne donnent des indications valables qu'à condition de réaliser une longue suite d'essais divers.

L'analyse chromatographique en phase gazeuse fournit des renseignements très utiles mais incomplets.

En conclusion, l'auteur retient, comme test pratique, les essais comparatifs sur laits. A condition de disposer d'un alcool amylique valable, pris comme réactif de référence, l'épreuve de conformité peut se ramener à de simples déterminations acido-butyrométriques parallèles.

\section{Summary}

After making a review of the purity requirements applied in Belgium and other countries, the author comments the results of many controls of amyl alcohols for the Gerber fat determination.

The specific gravity's determination detects only the water and the inferior alcohols contents.

The distillation's curve is also chiefly influenced by those alcohols.

Distillation is very difficult to be realized correctly.

Preferably it can be replaced by gas-chromatographic estimation which gives more easily better indications. 
None of theses methods above mentioned detects the oily substances, furfural and other organic impurities.

Specific tests for these substances are often very difficult to be interpreted.

The blank test is no more satisfactory.

According to the author, only the comparative Gerber tests on milk are to be retained practically.

He proposes to specify as next the physico-chemical characteristics of the amyl alcohols for Gerber :

Amyl alcohol : specific gravity at $20^{\circ} \mathrm{C}: 0,81-0,82 \mathrm{~g} . / \mathrm{ml}$.

The amyl alcohol is to be composed from a chemically pure mixture of the two primary alcohols 3-methyl butanol-1 and 2-methyl butanol-1 (min. 95 per cent).

Other inferior alcohols as butanol-1 of 2-methyl propanol-1 may be present. (max. 2,5 per cent iso-butanol).

The quantity of impurities cannot be such as the amyl alcohol cannot fulfil the following suitability test :

\section{Suitability test :}

The fat contents of 3 milk samples with different contents ( $3 \pm 0,5$ per cent) are determined, according to Gerber, using on one side the amyl alcohol to be examined and on the other side a pure amyl alcohol, composed only from the two above mentioned primary alcohols. (eventually prepared by distillation).

The fat determinations are made in triplicate in selectioned butyrometers. (scale error $<0,03$ per cent).

The difference between the mean values of the nine in each of the series obtained results may not reach 0,03 per cent.

\section{BIBLIOGRAPHIE}

[1] Commission consultative des denrées alimentaires. Unification des méthodes d'analyse. - Bulletin de la Santé publique $n^{\circ} 1$ à 3, 1er trimestre 1949, p. 56.

[2] NBN 200. - Dosage de la matière grasse dans la crème. Méthode gravimétrique (Gerber-Roeder).

[3] NBN 209. - Dosage de la matière grasse dans la crème. Méthode volumétrique (Gerber-Koehler).

[4] NEN 962. - Bepaling van het vetgehalte van melk volgens de methode van Gerber. Nederlands Melk en Zuiveltijdschrift, vol. 18, n 4, p. 257.

[5] Milchw. Einheitsmethode, no 1, 1963. Fettbestimmung in Milch nach Gerber Milchwissenschaft 18, Heft 10, 1963, p. 514. 
[6] Normes françaises $V$ 04-214 (J. O. du 23 avril 1954). - Dosage de la matière grasse du lait par la méthode acido-butyrométrique de Gerber.

[7] 133 Beretning Hillerød. - Bestemmelse af fedtindholdet i maelk efter Gerber metode.

[8] 111 Beretning Hillerød. - 17 ärsberetning, 1956, 56-57, p. 145.

[9] 132 Beretning Hillerød. - 21 ärsberetning, 1960, 60-61, p. 140.

[10] Hänni et Ritter W. - Die Prüfung von Amylalkohol auf seine Zusammensetzung und Eigning als Reagenz zur Fett bestimmung nach Gerber. Milchwissenschaft 16, Heft 1, 1961, p. 24.

[11] Valiant et Herrington. - Amyl alcohol as a source of error in the Gerber test. Milchwissenschaft 14, Heft 6, 1959, p. 275. (15, 1960, p. 84.)

[12] Ersses. - Suggested specification for amyl alcohol (à paraître dans Nederlands Melk en Zuiveltijdschrift. Juillet 1965).

[13] Hingst et Thomasow. - Ueber die Prüfung von Amylalkohol. Milchwissenschaft, Heft 1, 1952, p. 16.

[14] Me Dowall. - The Buttermaker's Manual, vol. II, p. 1518. 